\title{
BMJ Open Quality Antibiotic stewardship in a tertiary care NICU of northern India: a quality improvement initiative
}

\author{
Sunita Agarwal, ${ }^{1}$ Jyoti Patodia (D) , ${ }^{2}$ Jaikrishan Mittal, ${ }^{2}$ Yatish Singh, ${ }^{2}$ \\ Vaibhav Agnihotri, ${ }^{2}$ Varun Sharma ${ }^{2}$
}

To cite: Agarwal S, Patodia J, Mittal J, et al. Antibiotic stewardship in a tertiary care NICU of northern India: a quality improvement initiative. BMJ Open Quality 2021;10:e001470. doi:10.1136/ bmjoq-2021-001470

- Additional supplemental material is published online only. To view, please visit the journal online (http://dx.doi.org/10. 1136/bmjoq-2021-001470).

Received 28 February 2021 Accepted 3 July 2021
Check for updates

(C) Author(s) (or their employer(s)) 2021. Re-use permitted under CC BY-NC. No commercial re-use. See rights and permissions. Published by BMJ.

${ }^{1}$ Department of Microbiology, SMS Medical College, Jaipur, Rajasthan, India

${ }^{2}$ Deparment of Neonatology, Neoclinic, Jaipur, Rajasthan, India

Correspondence to Dr Jyoti Patodia; jyoti_ag7@hotmail.com

\section{ABSTRACT}

Background The overuse of antibiotics in newborns leads to increased mortality and morbidities. Implementation of a successful antibiotic stewardship programme (ASP) is necessary to decrease inappropriate use of antibiotics and its adverse effects.

Problem Our neonatal intensive care unit (NICU) is a tertiary referral centre of north India, consisting of all outborn babies mostly with sepsis caused by high rate of multidrug-resistant organisms (MDROs). So antibiotics are not only life-saving but also used excessively with a high antibiotic usage rate (AUR) of 574 per 1000 patient days. Method A quality improvement (QI) study was conducted using the Plan-Do-Study-Act (PDSA) approach to reduce AUR by at least $20 \%$ from January 2019 to December 2020. Various strategies were made : such as making a unit protocol, education and awareness of NICU nurses and doctors, making check points for both starting and early stoppage of antibiotics, making specific protocol to start vancomycin, and reviewing yearly antibiotic policy as per antibiogram.

Results The total AUR, AUR (culture negative) and AUR (vancomycin) was reduced by $32 \%, 20 \%$ and $29 \%$, respectively, $(p<0.01)$. The proportion of newborns who never received antibiotics increased from $22 \%$ to $37 \%$ $(p<0.045)$ and the proportion of culture-negative/screennegative newborns where antibiotics were stopped within 48 hours increased from $16 \%$ to $54 \%(p<0.001)$. The compliance with the unit protocol in starting and upgrading antibiotic was $75 \%$ and $82 \%$, respectively. In early 2020 , there was a sudden upsurge in AUR due to central linerelated bloodstream infection breakout. However, we were able to control it, and all the PDSA cycles were reinforced. Finally, we could reattain our goals, and also able to sustain it until next 1 year. There was no significant difference in overall necrotising enterocolitis and mortality rates.

Conclusion In a centre such as ours, where sepsis is a leading cause of neonatal deaths, restricting antibiotic use is a huge challenge. However, we have demonstrated implementation of an efficient ASP with the help of a dedicated team and effective PDSA cycles. Also, we have emphasised the importance of sustainability in success of any Ql study.

\section{BACKGROUND}

Antibiotics are the most commonly used medication in neonatal intensive care unit
(NICU). Sepsis being the leading cause of mortality and morbidity, globally as well as in India, the treatment and survival of newborn infants, in particular the premature, is hugely dependent on effective antibiotics. ${ }^{12}$ A riskbased approach with low threshold is often used for starting antibiotic in neonatal sepsis, which has been quite successful in lowering its incidence, but increased number of noninfected infants are exposed to antibiotics. Empiric therapy is often extended to five to 5-7 days even in the absence of positive blood cultures. ${ }^{3}$ Infants commonly present with non-specific systemic signs suggestive of infection, leading to the frequent use of broadspectrum empirical antibiotics in infants who are subsequently found to be uninfected. Unreliable clinical signs, disastrous outcome in case of delayed start of antibiotic treatment and reluctance to withdraw initiated treatment often result in overuse of antibiotics in NICU.

Antibiotics are powerful, life-saving drugs, but when used inappropriately, they may have serious adverse effects. Prolonged empirical antibiotic use among preterm infants with negative cultures has been associated with an increased risk of mortality and morbidities such as late-onset sepsis (LOS), necrotising enterocolitis (NEC), >stage 3 retinopathy of prematurity, emergence of fungal infections and multidrug-resistant organisms (MDRO), and also poor neurodevelopmental outcomes. ${ }^{4}$ Antibiotic overuse causes disruption of the microbiome, which may have lasting consequences reflected as dysbiosis, increased carriage of antibiotic resistance genes and MDROs. Each additional day of antibiotic exposure in the absence of positive blood cultures increases the risk of NEC in very low birthweight (VLBW) babies $7 \%-20 \% .^{5}$

In a recent study, Flannery et at demonstrated that $78.6 \%$ of VLBW and $87 \%$ of extremely low birthweight (ELBW) infants 
were treated with antibiotics in their first days of life. ${ }^{6}$ Additionally, as per a meta-analysis, $26.5 \%$ of VLBW and $37.8 \%$ of ELBW infants received more than 5 days of antibiotic treatment. ${ }^{7}$

One of the most effective measures to reduce unnecessary antibiotic exposure and its adverse outcomes is by implementation of antibiotic stewardship programmes (ASPs). The WHO identified the development of a national and institutional antibiotic stewardship programmes (ASPs) as a key instrument to tackle this concern. ${ }^{8}$ This has prompted calls in the USA and the UK for national action plans to combat antibiotic resistance. ${ }^{9}$ CDC (Centers for Disease Control and Prevention) launched a collaborative QIP (Quality Improvement Programme) with VON (Vermont Oxford Network), the world's largest neonatal benchmarking organisation. ${ }^{10}$

However, unlike the paediatric ASP, which has proven to be effective, lack of evidence-based strategies and easyto-use guidelines at the point of care preclude adoption of best practices for the use of antibiotics in neonates. ${ }^{11}$ There is lack of a validated antimicrobial guideline that addresses the unique challenges of the NICU environment, such as culture-negative clinical sepsis and empirical treatment of early-onset sepsis (EOS).

\section{PROBLEM}

Our NICU is a tertiary referral centre of north India, Rajasthan. It consists of only outborn babies with around 1200 neonates admitted every year. Most of the babies referred are sick with severe sepsis. We have a high culture-positive sepsis rate of around $14 \%$. Also, we have many surgical patients $(5 \%)$ and $15 \%-20 \%$ of our babies are VLBW babies. We empirically have to start most of our babies on broad-spectrum antibiotics, as they are life-saving. As newborns often present with non-specific systemic signs suggestive of infection, we end up using broad-spectrum empirical antibiotics in infants who are subsequently found to be uninfected. Also, most of the babies referred to us are already on broad-spectrum antibiotics as often in the periphery there is a tendency to start multiple antibiotics for sepsis. It was seen that NICU in the low resource settings referring the babies to our centre had complete lack of awareness about the growing emergence of MDRO and other adverse effects of unnecessary and prolonged antibiotics use. Also, antibiotic sensitivity trend at our centre over the years has shown alarming results. There has been a drastic increase in MDRO, especially, carbapenem-resistant Gram-negative bacilli (GNB: Klebsiella), which was the most common organism leading to sepsis, with sensitivity to meropenem only in 20\%-30\%. Other resistant organisms reported at our centre were coagulase-negative Staphylococci (CONS; $5 \%$ ), methicillin-resistant Staphylococcus aureus (MRSA: 7\%), and vancomycin-resistant Enterococcus (VRE). At the same time, MDRO were leading to fulminant sepsis, being the most common cause of mortality at our centre. As our centre is a referral centre catering sick babies all over from north India, this pattern of emerging antibiotic resistance actually represents the current scenario in India which is in itself is a medical emergency. The only solution to this was implementing an effective ASP in our NICU to promote judicious antimicrobial use and control the emergence of MDROs. Many quality improvement (QI) projects have demonstrated success with implementation of ASP. However, more information is needed to identify additional strategies to safely reduce antibiotic use in the NICU in an outborn centre such as ours, which contains predominantly septic babies with high rate of MDROs. It would be a great challenge for us to restrict antibiotic use without compromising the safety of our patients.

\section{METHODOLOGY}

Study design

A QI study using WHO Point of Care Quality Improvement Model ${ }^{12}$ using Plan-Do-Study-Act (PDSA) cycles approach, was planned to implement ASP in our NICU. Our main aim was to reduce excessive and inappropriate antibiotic use in NICU to prevent emergence of MDROs over a span of 2 years (January 2019 to December 2020). This QI study was carried out in a Tertiary care referral centre, Neoclinic Hospital, Jaipur, which is one of the largest 75 bedded level III NICU in Rajasthan, with around 1200 NICU admissions/year. All preterm and term newborns admitted to our NICU (in the first 28 days of life) were included in the QI study. All the babies admitted in our NICU are outborn.

\section{MEASURES}

Our primary outcome measure was to decrease antibiotic usage rate (AUR) by at least $20 \%$ from baseline. AUR was calculated separately as total AUR, AUR in culture negative and AUR for vancomycin.

AUR $/ 1000$ patient days $=($ total days of any antibiotic use /total days of admission) $\times 1000$.

Administration of a single antibiotic for a day is considered as 1 day, irrespective of dosage strength or number of doses administered per day. For example: administration of meropenem as a single dose of $20 \mathrm{mg} / \mathrm{kg}$ or 40 $\mathrm{mg} / \mathrm{kg}$ or three times a day ( 8 hourly) would be considered as 1 day of antibiotic use. A single patient receiving both vancomycin and meropenem on the same day would be considered as 2 days of antibiotic.

- AUR in culture negative $/ 1000$ patient days= (total days of antibiotic use in culture negative/total days of admission) $\times 1000$.

- AUR for vancomycin/1000 patient days= (total days of vancomycin use/total days of admission) $\times 1000$.

Other process measures included in our study were:

- Proportion of neonates not exposed to antibiotics= (number of neonates that never received any antibiotic during stay/number of neonates admitted) $\times 100$.

- Compliance with unit protocol in starting antibiotics $=$ (number of times antibiotics started 
appropriately as per protocol/number of times antibiotics prescribed) $\times 100$.

- Compliance with unit protocol in upgrading antibiotics $=$ (number of times antibiotics upgraded appropriately as per protocol/number of times antibiotics prescribed) $\times 100$.

- Early stoppage of antibiotics:

Proportion of culture-negative and sepsis screennegative patients where antibiotics would be stopped appropriately at 48 hours was calculated $=$ (number of times antibiotics were stopped at 48 hours/number of times antibiotic prescribed in culture negative and screen negative) $\times 100$.

- Prolonged antibiotic use: Prolonged antibiotic use was defined as antibiotic duration more than 48 hours in screen negative and culture negative, more than 7 days in screen positive and culture negative, more than 14 days in culture positive and more than 21 days in cases with meningitis.

In addition, we also measured other complications associated with antibiotic overuse, such as NEC and overall mortality rates, using data from the admission register and medical records.

Our study was divided into three phases: initial observation phase (12 weeks), implementation phase (five PDSA cycles: 10 months) and postimplementation phase/ sustainability (10 months: to be continued).

Observation phase: Baseline data were collected on predesigned proforma at our centre between January 2019 and March 2019 (12 weeks). In our baseline data, it was seen that total AUR was 574, AUR in culture-negative babies was 451 and AUR of vancomycin was 62/1000 patient days. Overall, $22 \%$ of newborns were not exposed to antibiotics during NICU stay. Antibiotics were stopped within 48 hours in culture-negative and screen-negative patients in only $16 \%$ of cases, whereas in culture negative but screen-positive patients antibiotic stopped at 7 days in $54 \%$. Overall, $28 \%$ of the babies received prolonged antibiotics.

We further did a root cause analysis of our problem and identified various factors which were contributing to unrestricted antibiotic use in our babies, such as: lack of a unit antibiotic protocol, lack of antibiotic policy based on antibiogram, lack of motivation and awareness regarding burden of antimicrobial resistance among healthcare personnel, lack of knowledge about when to start and stop antibiotics appropriately.

A multidisciplinary antibiotic stewardship QI team was formed comprising two neonatologists, a microbiologist, nursing-in-charge, four nursing staff and a fellow student. All the team members were assigned a specific duty. The team members worked together to evaluate factors contributing to overuse of antibiotics, and further plan and implement strategies to reduce the inappropriate use of antibiotics (AUR/1000 patient days) in NICU by at least by $20 \%$.

\section{Strategy}

Strategies were made in the form of PDSA cycles to implement ASP in the NICU.

1. First PDSA cycle: (April 2019 to May 2019). Plan: Formulation of unit sepsis and antibiotic protocol, as there was no specific unit protocol for antibiotic prescription. Do: A meeting was held and adaptation of The National Institute for Health and Care Excellence guidelines, $2016^{13}$ for EOS and an adaption of neonatal and paediatric sepsis ${ }^{14}$ guidelines for LOS was done to ensure uniformity of antibiotic prescription (refer: online supplemental file 1). Educational interventions including presentations and posters (refer : online supplemental file 2) outlining sepsis guidelines, data from the baseline period and information about antibiotic abuse including emerging antibiotic resistance were disseminated in the unit. A sheet of protocol was attached to every patient's file so as to review the actual need to start, stop, upgrade or continue antibiotics as per protocol. Study: Baseline AUR decreased from 574 to 457 per 1000 patient days. Act: This PDSA cycle was adopted and continued.

2. Second PDSA cycle: (June 2019 to July 2019). Plan: Early stoppage of antibiotics in non-septic babies. Do: A mandatory checkpoint was made at 48 hours of starting antibiotics. If blood culture was negative and 2 CRP were negative 24 hours apart, the patient being asymptomatic, then antibiotics would be stopped. Similar checkpoint was made at 7 th day, and antibiotics in culture-negative and screen-positive patients were not given for more than 7 days. Study: AUR decreased from 457 to 431 per 1000 patient day. Act: This cycle was adopted and continued.

3. Third PDSA cycle: (August 2019 to September 2019). Plan: Restriction of antibiotic initiation. Do: Before any baby is started on antibiotics, it was reviewed by the consultant in NICU, and antibiotics were started only as per the protocol. Study: AUR decreased from 431 to 426 per 1000 patient days. Act: This cycle was adopted and continued.

4. Fourth PDSA cycle: (November 2019 to December 2019). Plan: Formulation and implementation of new antibiotic policy as per antibiogram. Do: An antibiogram was made, after reviewing the sepsis and antibiotic sensitivity pattern of last 1 year, and first, second and third-line antibiotic were revised, and a poster regarding it was put in all NICUs. Study: Here, there was increase in AUR from 426 to 443. The reason for this was central line-related bloodstream infection (CLABSI) breakout in the unit and also change of fellow residents due to training session changeover. Act: The cycle was adapted and along with that a senior neonatologist in the unit was given the responsibility of ASP and formation of antibiogram and sepsis control measures, so that the project would remain unaffected by change of fellow residents.

5. Fifth PDSA cycle: (January 2020 to February 2020). Plan: Reducing the use of vancomycin (AUR). Do: A 
meeting was held, and a written protocol was made for starting and stopping of vancomycin in NICU, which was circulated among all the staff and doctors (refer online supplemental file 3). Study: Vancomycin AUR decreased from 54 to 37 per 1000 patient days, and overall AUR decreased from 443 to 411 per 1000 patient days. Act: This cycle was adopted and continued.

Post Implementaion phase: Sustaining QI initiatives (March 2020 to December 2020).

Sustaining an improvement is a must and for this the QI team met weekly to discuss the various PDSA cycles and review the antibiotic usage data. A regular monitoring of AUR and other measures was done via a trend line, a checklist to ensure compliance with the unit protocol, and a regular audit by feedback system in monthly statistical meet was held. During this QI study, there were no major costs incurred and no additional staff were recruited.

\section{STATISTICAL ANALYSIS}

Continuous analysis was done on statistical process control (SPC) charts to evaluate the trend of AUR, and the process measures. Preliminary analysis was expressed as mean (SD), percentage and frequencies. For parametric data, $\chi^{2} /$ Fisher exact test and for continuous variables t-test were used. Comparison between observation and implementation phase was done in terms of baseline characteristics, various interventions and complications of antibiotic abuse. The $p$ value of less than 0.05 was taken as significant. Analysis was done using the SPSS V.20.0 for Windows (IBM Corporation).

\section{RESULTS}

The QI study involved around 2292 newborns over a period of 2 years; 290 in observation phase (January to March 2019), 1138 in intervention phase (PDSA cycles: April 2019 to February 2020) and 864 in sustainability phase (March to December 2020).

We studied the baseline characteristics of newborns enrolled in the study in terms of gender, birth weight and gestational age which was found to be similar in observation, intervention and sustainability phase. During the study, $73.1 \%$ were males and $26.9 \%$ were females. The mean gestational age was 34 weeks \pm 4.45 , and mean birth weight was $2017 \pm 947 \mathrm{~g}$ (refer:online supplemental file 4).

Our primary outcome: AUR is our primary outcome and its trend throughout the study has been depicted in figure 1. It presents an SPC chart, U type, which is a

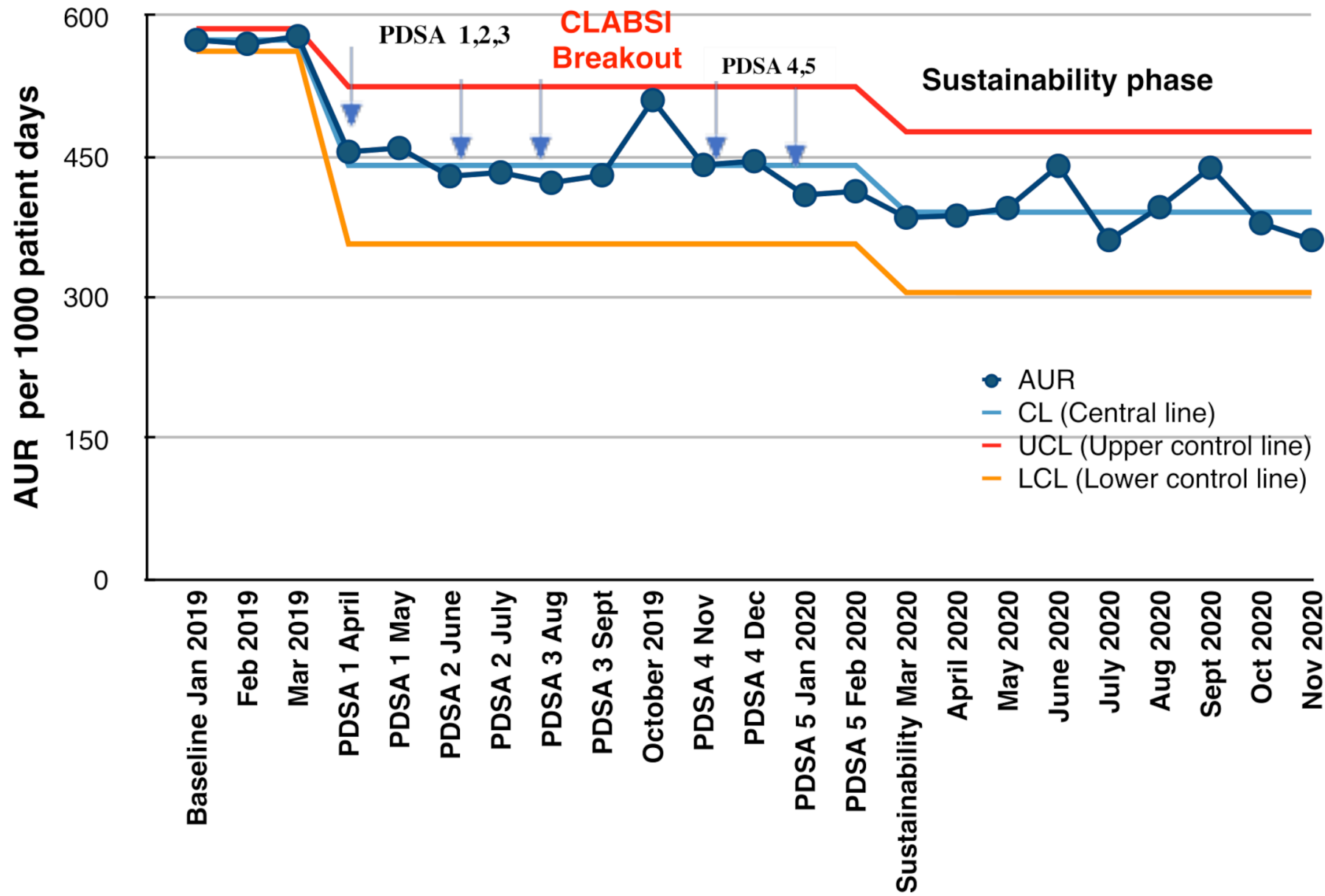

Figure 1 Statistical process control (U-chart) showing monthly antibiotic usage rate (AUR) and the various Plan-Do-StudyAct (PDSA) cycles during the antibiotic stewardship quality improvement programme from January 2019 to December 2020. CLABSI, central line-related bloodstream infection. 
time ordered graphical representation of a process, used to determine if a process has been operating in statistical control, helps in maintaining it, and determine any common cause or special cause variation if any. Here, central line (CL) is calculated as mean AUR of the observation period, upper control line (UCL) is calculated as mean+3 SD and lower control line (LCL) is calculated as mean-3 SD. CL has been adjusted two times as special cause variation was identified on two occasions, one is soon after the implementation of PDSA one itself,that is, implementation of unit sepsis and antibiotic protocol after which there has been sudden fall in AUR by almost $20 \%$ crossing the LCL, and the other is after the CLABSI breakout in October where there was sudden increase in AUR touching the UCL.

There was a significant fall in AUR from 574 to 457 per 1000 patient days (almost by 20\%) after the first PDSA cycle itself, after which there was only mild variation in AUR reflecting common cause variation. But after the third PDSA, during the month of October 2019, there was sudden spike in AUR upto 510 per 1000 patient days which coincided with CLABSI breakout in NICU. We have shown the trend of monthly AUR in each PDSA cycle (figure 1). This spike was mainly due to two reasons; one of the fellow residents who was primarily involved in this QI study completed his term and left the hospital, and a new fellow had just joined who was handed over the QI project. The second reason was there was a CLABSI breakout in the NICU (five CLABSI in 4 weeks). So to control the situation, a senior consultant was permanently given the responsibility of the ASP programme, so that it would be unaffected by the new trainees joining every year. Epidemiological and microbiological surveillance showed the emergence of MDROs in our unit, so we planned our next PDSA cycle accordingly. We made an antibiogram of last 1 year and revised our antibiotic policy accordingly. So, we introduced new PDSA cycles 4 and 5 and reinforced all the previous cycles. Hence, with appropriate clinical management and strengthening infection prevention and control measures, we could control the spike of AUR. By the next month (November 2019), itself AUR came back to its baseline and further decreased which was sustained for at least 10 months. Overall, AUR was decreased from 574 to 390 per 1000 patient days, which was almost reduced by $32 \%(\mathrm{p}=0.001)$. This was more than what we had targeted to reduce AUR by at least $20 \%$.

We also measured AUR in culture-negative patients, which decreased from 451 to 361 per 1000 patient days, that is, reduced by $20 \%(\mathrm{p}=0.015)$. As vancomycin was being used quite frequently in our unit, we measured AUR for vancomycin, which was 62/1000 patient days, and there was no reduction in its use initially, so a separate fifth PDSA cycle was introduced, where a protocol for starting vancomycin was introduced. After this, vancomycin usage rate decreased significantly upto 44/1000 patient days, which is almost $29 \%$ reduction $(\mathrm{p}=0.03)$ (refer table 1).
We measured many other process indicators that would indicate towards judicious use of antibiotics (table 1). One of these most important was percentage of babies not exposed to antibiotics at all during NICU stay, which was initially $22 \%$ which showed good increase from first PDSA cycle itself and gradually increased to $37 \%$ in sustainability phase $(p=0.045)$. We also measured compliance with our new protocol during initiation and upgradation of antibiotics, which was $60 \%$ and $54 \%$, respectively, during the first PDSA cycle, and it increased upto $75 \%$ and $82 \%$ in the sustainability phase $(p<0.05)$. We measured early stopping of antibiotics at 48 hours in culture-negative and sepsis screen-negative patients and stopping of antibiotics at 7 days in culture negative but sepsis screen-positive patients, which was only $16 \%$ and $54 \%$, respectively, initially. After introduction of first PDSA cycle itself that focused on early stoppage of antibiotics, it increased drastically and was maintained in sustainability phase upto $54 \%(\mathrm{p}<0.000)$. Also, prolonged use of antibiotics decreased from $28 \%$ to $20 \%(\mathrm{p}<0.038)$.

In October 2019, during CLABSI breakout and change of fellow student in the NICU, with increase in AUR, there was also increase in use of prolonged antibiotic (26\%), percentage of babies not exposed to newborn was decreased (30\%), compliance to protocol for starting antibiotics remained $70 \%$ and antibiotic stopped early at 48 hours in $44 \%$ (as shown in figure 2). The best part was, we demonstrated a sustainability phase of 10 months where all are targets were achieved and also maintained. We also looked into overall NEC and mortality rates after starting the QI study. NEC rates decreased slightly from $7.3 \%$ (2018) to 5.7\% (2020); however, it was not statistically significant $(\mathrm{p}=0.56)$, whereas the mortality rate was almost similar: $6.8 \%$ in 2018 and $6.5 \%$ in $2020(\mathrm{p}=0.87)$.

\section{DISCUSSION}

A multidisciplinary QI initiative was taken to ensure judicious antibiotic use at our NICU. This was the first time we initiated antibiotic stewardship in our hospital. Reviewing the usage of antibiotics at the NICU helped us identify the various barriers for safe antibiotics prescriptions. Further, PDSA cycles helped us to implement the solutions, which were effective and efficient.

Overall, our QI approach was found to be quite effective, as we were able to reduce overall AUR from 574/1000 patient days to $390 / 1000$ patient days while in culturenegative patients AUR reduced from 451/1000 patient days to $361 / 1000$ patient days. An overall decrease of $32 \%$ in overall AUR and 20\% in AUR in culture-negative patients was achieved against a target of $20 \%$. Our study showed a comparatively higher AUR, which could be because of the fact that all babies in our NICU are outborn and referred cases with high rate of sepsis. So we could bring our AUR down mainly by decreasing antibiotic usage in culture-negative babies, as culturepositive babies would require appropriate antibiotics with adequate duration. However, it was seen that our 


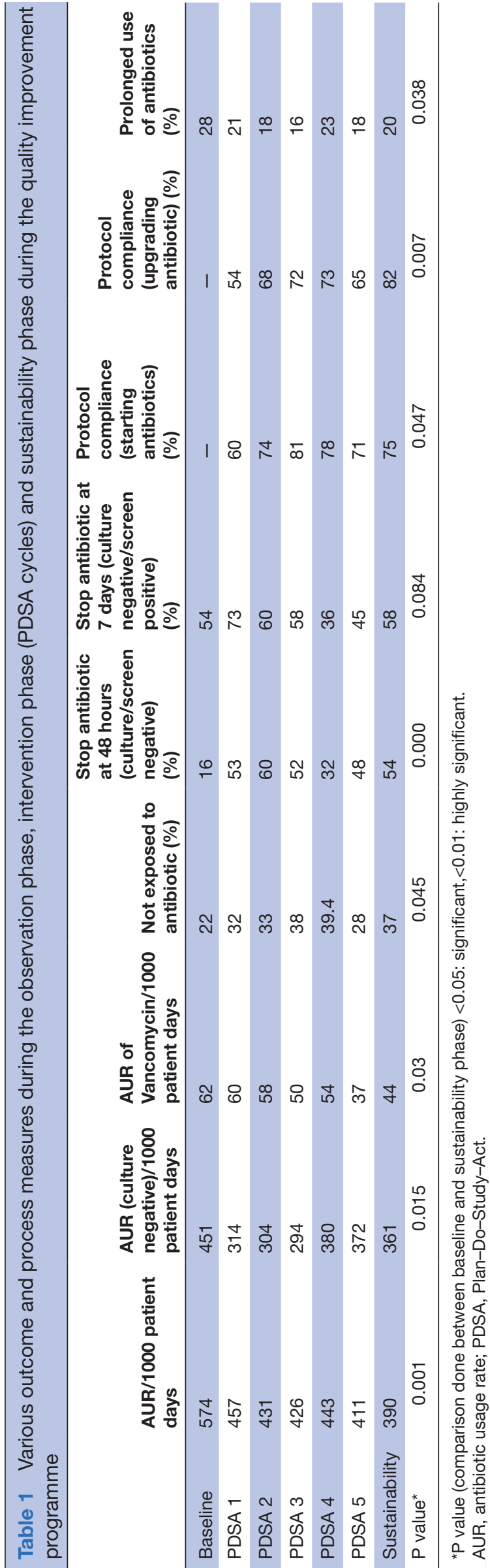

AUR in culture negative decreased only by $20 \%$ and in total by $32 \%$. So we can focus more on decreasing antibiotic usage in culture-negative patients. For this, we had introduced two PDSA cycles, that is, first, restriction in initiation of antibiotics by having a strict compliance to unit protocol and second, by early stoppage of antibiotics by making mandatory checkpoints at 48 hours for culture-negative/screen-negative and at 7 days for culture-negative and screen-positive patients that helped us in decreasing the prolonged use of antibiotics. There was an increase in compliance for initiation and upgradation of antibiotics after each PDSA cycle, increasing from initial $60 \%$ to $75 \%$ and from $54 \%$ to $82 \%$, respectively. We were able to increase early discontinuation of antibiotics at 48 hours from $16 \%$ to $54 \%$ over the study period and decrease prolonged use of antibiotics from $28 \%$ to $20 \%$. The proportion of newborns never exposed to antibiotics increased from $22 \%$ to $37 \%$.

Similar to our study, Lu et al also concluded that the ASP was feasible and effective in reducing the AUR by $30 \%$ among the neonates in a predominantly outborn tertiary centre. The proportion of infants colonised with MDRO during the study decreased from $1.4 \%$ to $1.0 \%$ post intervention. The safety matrices such as readmission for sepsis $(1.2 \%$ vs $1.1 \%)$ and sepsis-related mortalities $(0.24 \%$ vs $0.23 \%)$ did not show significant changes over time. ${ }^{15}$ Thus, the ASP was effective in reducing antibiotic exposure without affecting the quality of care. We also need to sustain our study and continue it further to see significant effect on long term outcomes such as rates of MDROs. We did evaluate the overall NEC and mortality rates during our study, which were similar and there was no decrease in NEC and mortality rates. May be we need to sustain the study further, and further improve our outcome and other process measures to bring significant effect on mortality and NEC rates. Also, there are many other factors that have an impact on NEC and mortality which need to be kept in mind.

One of the common causes of LOS in newborns is CONS species, MRSA, VRE and extended-spectrum betalactamase producing organisms, which is often resistant to all beta-lactam antibiotics, requiring treatment with vancomycin. ${ }^{16}$ Thus, vancomycin is a frequently used antibiotics for suspected LOS in the NICU. Most common infection in our NICU is GNB sepsis (Klebsiella, 60\%), out of which almost $50 \%$ is carbapenem (meropenem) resistant, which is our second-line antibiotic. So, whenever we suspect GNB sepsis or blood culture is appearing to be positive, we upgrade the antibiotic to our third-line antibiotics, which is colistin as per the unit antibiogram. Gram-positive cocci such as CONS and MRSA are the second most common cause of sepsis in the unit, which is mostly $(>80 \%)$ sensitive to most of the antibiotics. It was observed that in our unit whenever we suspected ventilator-associated pneumonia (VAP) or CLABSI or postoperative, often vancomycin was started empirically. Also, in last few years, we have started encountering cases of VRE (2-3/year). So, we noticed that we could avoid 


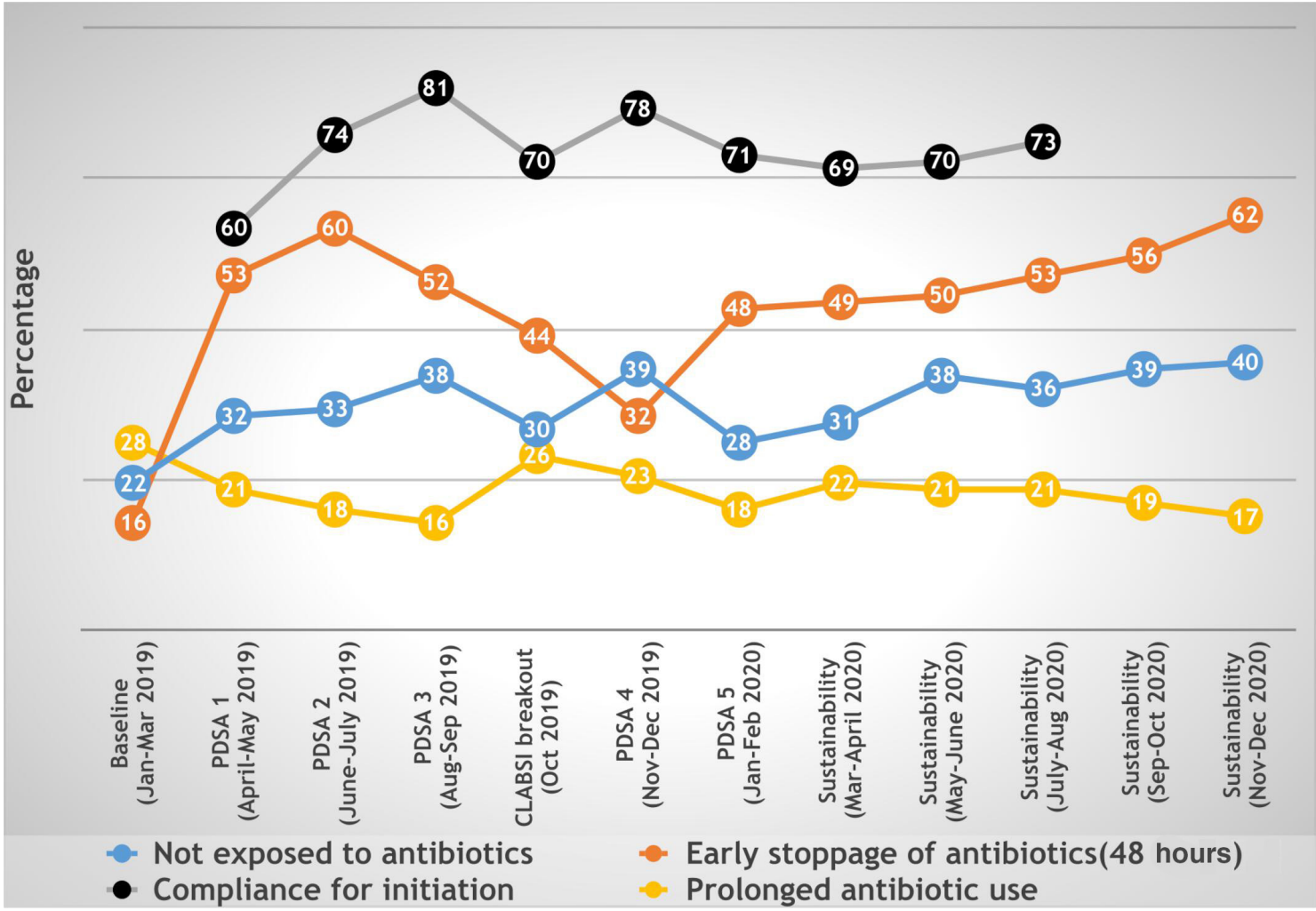

Figure 2 Time series graph showing various process measures of antibiotic usage during the antibiotic stewardship quality improvement programme from January 2019 to December 2020. PDSA, Plan-Do-Study-Act.

using vancomycin, and instead use drugs that act on both gram negative as well as Gram-positive organisms. So, we planned to specifically decrease the use of vancomycin, as this is an essential antibiotic which should be reserved for future use, as it would not be possible for us to decrease the use of other antibiotics such as meropenem, gentamicin, amikacin, colistin significantly as per our organisms and it's sensitivity pattern. Thus, including a protocol to restrict the use of vancomycin in NICU in our ASPs could help us avoid emergence of antibiotic-resistant organisms. Hence, we introduced the fifth PDSA cycle to decrease vancomycin usage, in which we were successful in decreasing AUR of vancomycin by $29 \%$. In a similar study by Chiu et al, vancomycin starting rates were reduced from 6.9 to 4.5 per 1000 patient days $(35 \%$ reduction; $\mathrm{p} 0.01) .{ }^{17}$ Implementation of an NICU vancomycin use guideline significantly reduced exposure of newborns to vancomycin without adversely affecting short-term patient safety. Further studies are required to evaluate the long-term effect of vancomycin restriction on NICU patient safety, particularly among institutions with higher rates of MRSA infections.

\section{CHALLENGES WE FACED}

The initial challenge was to take risks of deviating from the usual practice of starting antibiotics in most of the babies by the NICU doctors in fear of adverse clinical incidents. This was managed by bringing awareness among the doctors and staff especially about the alarming situation of emerging antibiotic resistance and its adverse outcomes, this motivated all to decrease antibiotic usage. As our centre is a referral centre, we have babies being referred from all over Rajasthan, which are quite sick and predominantly septic or requiring surgery. Often it becomes quite difficult to differentiate between septic and non-septic babies as signs and symptoms in newborns are very non-specific. So, there is tendency of adding antibiotics to most of the babies in fear of any clinical deterioration. Also, many babies are coming with sepsis via $\mathrm{MDRO}$, and are already on broad-spectrum multiple antibiotics when referred to our centre, so the decision to stop or even downgrade antibiotics could be quite risky. But, however, we have studied the antibiotic sensitivity pattern of our centre as well as many referring centres, and thus planned out antibiotics policy as per the sensitivity pattern, which was very helpful. Also, we focused more in decreasing AUR in culture-negative babies. Whenever we took the decision of stopping or not starting the antibiotic in a baby, it was followed by a very close monitoring of the babies with frequent reviews so that we could intervene as early as possible in case of any worsening. Also, we had a CLABSI break out in October 2019, where our ASP almost was on the verge of collapsing. All the improvement that was done so far, suddenly reached to the baseline antibiotic usage from where we started. But it was the motivation and confidence go the team leader that kept us going, and we learnt that success of any ASP also depend on maintaining asepsis in the unit 
and following CLABSI and VAP bundles simultaneously. Focussing on one programme does not mean we can ignore other important ongoing protocols in the NICU, especially, maintaining asepsis protocols.

\section{STRENGTHS}

Our enthusiastic and dedicated team was our main strength who worked passionately to decrease AUR in the NICU. We have successfully demostrated decrease in antibiotic usage by an effective QIP using simple and effective PDSA cycles. The emerging antibiotic resistance pattern seen in our NICU also represents the sensitivity pattern of the rest of Rajasthan from where babies are referred to us. As we take blood cultures of all babies at admission that represents the sepsis profile of the referral centres. For example, most common organism causing sepsis was found to be Klebsiella both at our centre and rest of the referral centre, and Klebsiella at our centre had only $15 \%$ sensitivity to meropenem whereas in the periphery its sensitivity was $30 \%$ which is also quite low. Hence, there is an urgent need of ASP in most of the NICUs around in North India and the best way to implement it would be through a QI programme, similar to ours. The strategies and PDSA cycles used by us are simple enough which, can be followed by other NICUs as well. Hence, our study and its methodology can be generalised to most of NICUs of north India of similar settings. Also, the study did not add any extra cost, manpower, or equipments, hence it can be replicated even in resource limited settings. We have not only been able to achieve our goals in terms of decreasing AUR, but also able to sustain it. We have demonstrated a sustainability of around 10 months by frequent reviews of AUR, monthly sepsis meet and direct involvement of a senior neonatologist and a microbiologist in leading the entire ASP. We did weekly reviews, motivating the team to check antibiotic use, along with lectures, poster and study material, so that staff remained motivated and confident throughout.

\section{LIMITATIONS}

One of the major limitation of our study was that all our babies are outborn and most of the babies referred to us were already on antibiotics and also received antibiotic outside. We did not take this into account as it was not possible for us to control it. This could have effected the impact of our QI study and may be we could have shown better improvement if our babies were inborn. Often, data from different neonatal units may not be comparable directly owing to the differences in admission populations, baseline rates of sepsis, and variations in practices. But as ours is a referral centre, we assume that our data and research outcome are generalisable to other NICUs.

\section{CONCLUSION}

ASP may be implemented in all NICUs to decrease inappropriate antibiotic usage, and QI initiative is an effective way of doing it. Introducing a unit protocol for sepsis/ antibiotic policy as per antibiogram and making mandatory checkpoints at 48 hours to stop antibiotic in culturenegative sepsis were the most effective PDSA cycles. Maintaining sustainability is the key to success of any QI programme as demonstrated by our study. We are further motivated to continue with our QI programme so that we can see if there is any effect on rates of MDRO. Also, our study is applicable to other NICUs of similar settings. Infact we look forward to motivate and sensitise our referring centres to start an ASP by making them aware of the emerging resistance pattern of microbes at their centre.

Acknowledgements The authors acknowledge neonatal intensive care unit team (doctors and nurses).

Contributors SA and JP designed the study and drafted the manuscript. YS and VA were involved in data collection. VS and JP did data analysis, JM critically reviewed it.

Funding The authors have not declared a specific grant for this research from any funding agency in the public, commercial or not-for-profit sectors. Publication of this article is made Open Access with funding from the Nationwide Quality of Care Network.

Competing interests None declared.

Patient consent for publication Not required.

Ethics approval This study was done as a part of quality improvement initiative in our hospital, and informal consent was taken from all parents/guardians before participation.

Provenance and peer review Not commissioned; externally peer reviewed.

Data availability statement All data relevant to the study are included in the article or uploaded as supplementary information.

Supplemental material This content has been supplied by the author(s). It has not been vetted by BMJ Publishing Group Limited (BMJ) and may not have been peer-reviewed. Any opinions or recommendations discussed are solely those of the author(s) and are not endorsed by BMJ. BMJ disclaims all liability and responsibility arising from any reliance placed on the content. Where the content includes any translated material, BMJ does not warrant the accuracy and reliability of the translations (including but not limited to local regulations, clinical guidelines, terminology, drug names and drug dosages), and is not responsible for any error and/or omissions arising from translation and adaptation or otherwise.

Open access This is an open access article distributed in accordance with the Creative Commons Attribution Non Commercial (CC BY-NC 4.0) license, which permits others to distribute, remix, adapt, build upon this work non-commercially, and license their derivative works on different terms, provided the original work is properly cited, appropriate credit is given, any changes made indicated, and the use is non-commercial. See: http://creativecommons.org/licenses/by-nc/4.0/.

ORCID iD

Jyoti Patodia http://orcid.org/0000-0002-1722-409X

\section{REFERENCES}

1 Astorga MC, Piscitello KJ, Menda N, et al. Antibiotic stewardship in the neonatal intensive care unit: effects of an automatic 48-hour antibiotic stop order on antibiotic use. J Pediatric Infect Dis Soc 2019;8:310-6.

2 Hsieh EM, Hornik CP, Clark RH, et al. Medication use in the neonatal intensive care unit. Am J Perinatol 2014;31:811-22.

3 Cantey JB, Wozniak PS, Pruszynski JE, et al. Reducing unnecessary antibiotic use in the neonatal intensive care unit (SCOUT): a prospective interrupted time-series study. Lancet Infect Dis 2016;16:1178-84.

4 Ting JY, Synnes A, Roberts A, et al. Association between antibiotic use and neonatal mortality and morbidities in very low-birth-weight infants without culture-proven sepsis or necrotizing enterocolitis. JAMA Pediatr 2016;170:1181-7. 
5 Cantey JB, Pyle AK, Wozniak PS, et al. Early antibiotic exposure and adverse outcomes in preterm, very low birth weight infants. $J$ Pediatr 2018;203:62-7.

6 Flannery DD, Ross RK, Mukhopadhyay S, et al. Temporal trends and center variation in early antibiotic use among premature infants. JAMA Netw Open 2018;1:e180164.

7 Rajar P, Saugstad OD, Berild D, et al. Antibiotic stewardship in premature infants: a systematic review. Neonatology 2020;117:673-86.

8 World Health Organization. Global action on antimicrobial resistance. Geneva: WHO Press, 2015.

9 UK Department of Health. Uk five year antimicrobial resistance strategy 2013 to 2018. London, UK: Department of Health, 2013.

10 The White House. National strategy for combating antibiotic resistant bacteria. Washington, D.C: The White House, 2014

11 Potocki M, Goette J, Szucs TD, et al. Prospective survey of antibiotic utilization in pediatric hospitalized patients to identify targets for improvement of prescription. Infection 2003;31:398-403.

12 World Health Organization. Learner's Manual-POCQI. NewDelhi,India: WHO, Regional Office for South-East Asia, 2017. http:// www.newbornwhocc.org/POCQI-Learner-Facilitator.pdf
13 NICE clinical guideline. Sepsis: recognition, diagnosis and early management; Antibiotic treatment in people with suspected sepsis[Internet]. London,UK: NICE (The National Institute for Health and Care Excellence), 2016. Available: https://www.nice.org.uk/ guidance/NG51 [Accessed 29 Nov 2016].

14 European Medicines Agency (EMA). Report on the Expert Meeting on Neonatal and Paediatric Sepsis[Internet]. London : EMA, 2010. Available: https://www.ema.europa.eu/en/events/expert-meetingneonatal-paediatric-sepsis [Accessed 8 Jun 2010]

15 Lu C, Liu Q, Yuan H, et al. Implementation of the smart use of antibiotics program to reduce unnecessary antibiotic use in a neonatal ICU: a prospective interrupted time-series study in a developing country. Crit Care Med 2019;47:e1-7.

16 Venkatesh MP, Placencia F, Weisman LE. Coagulase-Negative staphylococcal infections in the neonate and child: an update. Semin Pediatr Infect Dis 2006;17:120-7.

17 Chiu C-H, Michelow IC, Cronin J, et al. Effectiveness of a guideline to reduce vancomycin use in the neonatal intensive care unit. Pediatr Infect Dis J 2011;30:273-8. 